\title{
Variable stars in young open star clusters
}

\author{
Sneh Lata*, Anil Kumar Pandey
}

Aryabhatta Research Institute of Observational Sciences, Manora Peak, Nainital 263002,

Uttarakhand, India

\begin{abstract}
We have carried out time series photometry of the some young open clusters NGC 1893, Be 59, NGC 7380, Stock 8 and NGC 281 to search for pre-main-sequence variable stars. We have detected several pre-mainsequence variables associated to the young open clusters with periods ranging from $\sim 0.1$ to $\sim 50$ days. These pre-main-sequence variables could be $\mathrm{T}$ Tauri stars. The association of these variable stars with the cluster has been established on the basis of two colour diagrams, a colour-magnitude diagram and spectral energy distributions. The brightness of classical T Tauri stars is found to vary with a larger amplitude in comparison to weak line $\mathrm{T}$ Tauri stars. It is found that the amplitude of pre-main-sequence variables decreases with increasing mass, which could be due to the dispersal of discs of massive stars. We have also detected several stars as main-sequence variables, which are classified either as slowly pulsating B stars, $\beta$ Cep stars, $\delta$ Scuti stars or members of a new class of variable stars.
\end{abstract}

\section{Introduction}

The study of variable stars in young open clusters is important to understand the stellar evolution and circumstellar disc properties. A young open cluster is a group of stars which are young (a few Myrs) and embedded within the gas and dust of the molecular cloud from which they were born. These objects provide an ideal environment to study variability because they harbour a sample of stars which have approximately similar ages but different masses. Their basic properties like distance, age and reddening help to find the nature of variable stars. They contain main-sequence (MS) stars from hot O/B to late A type stars. Young open clusters also contain pre-main-sequence (PMS) objects which are still contracting and moving towards the MS in the Hertzsprung-Russell (HR) diagram. Stars lying on the MS as well as those in the PMS region both show brightness variations with time. PMS objects are divided in two groups: T Tauri stars (TTSs) (mass $\leq 3 \mathrm{M}_{\odot}$ ) and Herbig Ae/Be stars (mass $>3 \mathrm{M}_{\odot}$ ). TTSs are characterized by emission lines and a near infrared (NIR) excess. They are further classified on the basis of the strength of the $\mathrm{H} \alpha$ emission line and their NIR excess, in two categories: classical TTSs (CTTSs) and weak line (WTTSs). CTTSs have strong $\mathrm{H} \alpha$ emission and NIR excess whereas WTTSs show weak emission at the wavelength of $\mathrm{H} \alpha$ and little or no NIR excess.

It is believed that variability in PMS objects is mainly associated to stellar activity like spots on the surface (Herbst et al. 1994; Scholz et al. 2009; Lata et al. 2011, 2012 and 2016). The PMS objects could also be eclipsing stars (if they had close a close companion) or pulsating stars (if they

*sneh@aries.res.in 
are situated within an instability strip). Whereas stellar pulsation is responsible for the brightness variation in MS stars (Moskalik \& Dziembowski 1992; Stankov \& Handler 2005). The details about the variability of PMS objects and MS stars can be found in Lata et al. (2011, 2012, 2014 and 2016).

This work presents variable stars detected in the cluster regions Be 59, NGC 1893, NGC 7380, Stock 8 and NGC 281. These young open clusters have been selected because they contain several PMS as well as O/B type stars (Sharma et al. 2007; Pandey et al. 2008; Jose et al. 2008; Baade 1983; Chen et al. 2011). Star formation and the PMS evolution are the least understood phases of stellar life cycles. The study of the rotation periods in TTSs could provide an extra clue to our understanding about star formation and the PMS evolution. Therefore, it has become an active area of research during the last decade. A larger size telescope is needed for the observations of PMS stars as we know that these PMS objects are faint and are surrounded by the gas and dust of their parent molecular cloud. We plan to observe some of the young open clusters with the 3.6-m Devasthal Optical Telescope (DOT), Aryabhatta Research Institute of Observational Sciences (ARIES), India.

\section{Observations and Data Reduction}

The photometric monitoring of the Be 59, NGC 1893 and NGC 281 region was carried out in the $V$ and $I$ band using a $2048 \times 2048$ CCD camera attached to the 1.04-m Sampurnanand Aryabhatta Research Institute of Observational Sciences (ARIES) telescope (for details see Lata et al. 2011, 2012, 2017). The observations of NGC 7380 have been taken using the $0.81-\mathrm{m} \mathrm{f} / 7$ Ritchey-Chretien Tenagara automated telescope in southern Arizona (Lata et al. 2016). The observations of the Stock 8 region were taken using the 2.4-m Thai National Telescope (TNT) of the Thai National Observatory located on one of the ridges ( $2457 \mathrm{~m}$ ) of Doi Inthanon, the highest peak in Thailand (Lata et al. 2017). The pre-processing and photometry of the images have been done using ray removal was performed using Image Reduction and Analysis Facility (IRAF) ${ }^{1}$ and DAOPHOT (Stetson 1987) respectively. We have done both aperture and PSF photometry. If stars are not substantially isolated in the observed field, PSF fitting gives better results.

\subsection{Variable Identification and period determination}

The DAOMATCH (Stetson 1992) routine of DAOPHOT was used to find the translation, rotation and scaling solutions between different photometry files, whereas DAOMASTER (Stetson 1992) has been used to match the point sources. From the output of DAOMASTER, we get corrected magnitudes listed in a .cor file. This file is further used to detect variable stars. The variables in the region of Be 59, NGC 1893, NGC 7380, Stock 8 and NGC 281 were detected either by the RMS dispersion technique or by inspecting their light curves (for details, see Lata et al. 2011, 2012 and 2016). Fig. 1 shows the real light curves of variable stars taken from Lata et al. (2016). The most likely period of the detected variables is derived using Lomb-Scargle periodogram (Lomb 1976; Scargle 1982) and is given on top of each panel in Fig. 2. Fig. 2 shows a sample of phased light curves for PMS variables (Lata et al. 2014).

\subsection{Membership}

In order to define the nature of variable stars, it is necessary to ascertain their association to the cluster. For this we have used a photometric criterion in the absence of kinematic data. In this method, the

\footnotetext{
${ }^{1}$ IRAF is distributed by the National Optical Astronomy Observatory, which is operated by the Association of Universities for Research in Astronomy (AURA) under cooperative agreement with the National Science Foundation.
} 

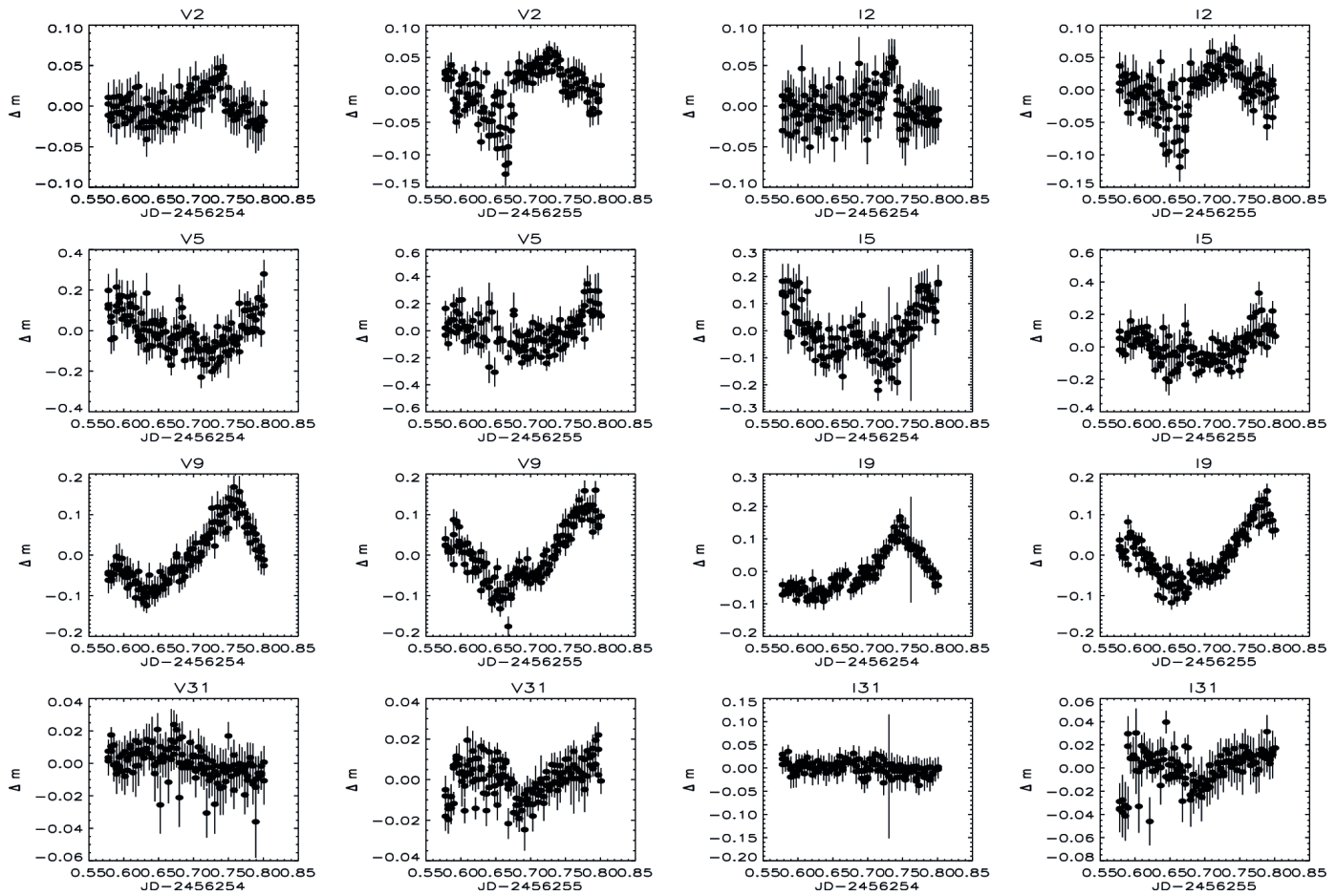

Figure 1: A sample of light curves of variable stars taken from Lata et al. (2016) in the cluster region NGC 7380.

$V / V-I$ colour-magnitude diagram (CMD), the $U-B / B-V$ two colour diagram (TCD) and the $J-H / H-K$ TCD were used to identify PMS and MS variables. We have also used spectral energy distributions (SEDs) to identify PMS variables and to study their circumstellar disc properties. For this purpose, we used models given by Robitaille et al. (2007).

\section{Characteristics of variable stars}

We have identified several MS variables in the cluster regions and these variables have been classified as $\beta$ Cep stars, slowly pulsating B (SPB) stars, $\delta$ Scuti variables, and a new class of variable stars. Their periods range from $\sim 0.1$ to $\sim 1$ days and their amplitudes lie in the range of $\sim 0.005$ to $\sim 0.2$ mag. The new class variables were first detected by Mowlavi et al. (2013) in the case of open cluster NGC 3766. In the H-R diagram these variables are found to be located between SPB stars and the $\delta$ Scuti stars, the region where no pulsations were expected on the basis of theoretical models. We have also detected several new class variables in the fields of NGC 1893 and NGC 7380.

The PMS variables detected in the cluster regions have masses $\leq 3 M_{\odot}$. Their masses and ages have been determined using the $V / V-I$ CMD. The ages of most PMS variables ranges from $\sim 0.1$ to $\sim 5$ Myrs. These PMS variables could therefore be TTSs. The estimated periods for the majority of these probable TTSs are in the range of $\sim 0.1$ to $\sim 50$ days, whereas their amplitude ranges from $\sim 0.005$ to $\sim 0.2 \mathrm{mag}$. The periodic variability in TTSs is believed to occur due to presence of either hot or cool spots on their surface (Herbst et al. 1994). We have found that CTTSs are varying with larger amplitude in comparison to the WTTSs, indicating the presence of hot spots on the surface of CTTSs. It is believed that hot spots form due to accretion of material from the circumstellar disc onto the stellar surface. Hence, a small fraction of the stellar surface is covered by these hot spots. As they have a high temperature, it causes brightness variations with a higher amplitude in CTTSs 

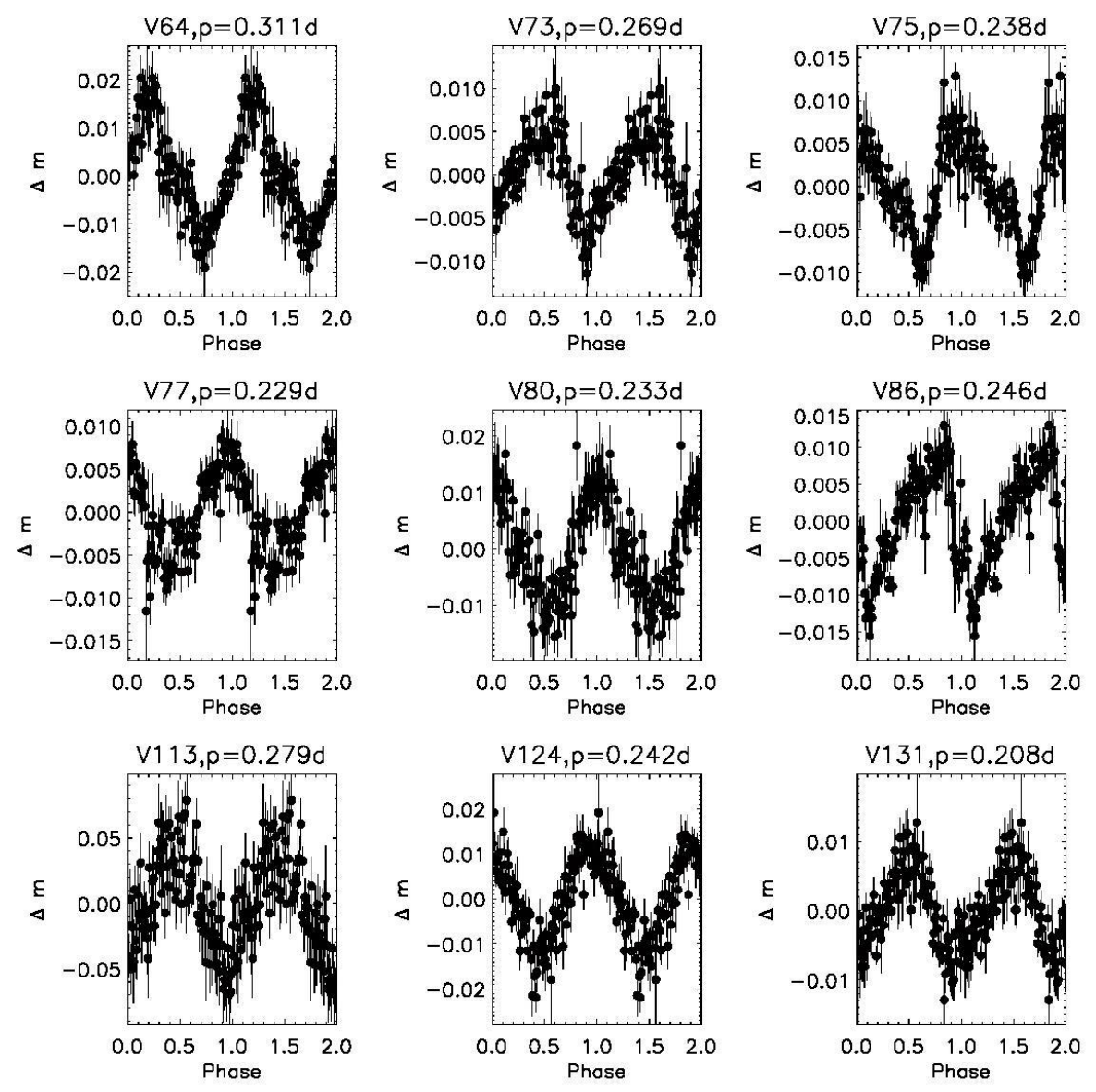

Figure 2: A sample of phased light curves for PMS variable stars in the cluster region NGC 1893. The data have been taken from Lata et al. (2014).

(Carpenter et al. 2001). The smaller amplitude in WTTSs suggests that these objects might have cool spots on their surface which are produced due to a magnetic field. It is found that the amplitude of TTSs decreases with increase in mass, which indicates the existence of a disc dispersal mechanism in relatively massive stars.

\section{Summary}

We have detected several PMS and MS variables in the cluster regions Be 59, NGC 1893, NGC 7380 and Stock 8 . The association of these variable stars with the cluster has been established on the basis of TCDs, CMD and SEDs. The MS variables are classified as $\beta$ Cep, SPB, $\delta$ Scuti stars and a new class of variable stars. The variables detected as PMS stars could be TTSs. The ages and masses of PMS variables have been determined using the $V / V-I$ CMD with the help of PMS evolutionary tracks (Siess et al. 2000). It is found that CTTSs are varying with a larger amplitude in comparison to WTTSs. We have also found that the amplitude of PMS variables decreases with increasing mass, which could be due to the dispersal of discs of relatively massive stars. 


\section{Acknowledgements}

We are thankful to the referee for a critical reading of the paper and useful comments.

\section{References}

Baade D. 1983, A\&AS, 51235

Chen W. P. et al. 2011, AJ, 142, 71

Carpenter J. M., Hillenbrand L. A., Skrutskie M. F. 2001, AJ , 121 , 3160

Herbst W., Herbst D. K., Grossman E. J., Weinstein D. 1994, AJ, 108, 1906

Jose J., Pandey A. K., Ojha D. K. et al. 2008, MNRAS, 384, 1675

Lata S., Pandey A. K., Maheswar G., Mondal S., Kumar B. 2011, MNRAS, 418, 1346

Lata S., Pandey A. K., Chen W. P., Maheswar G., Chauhan N. 2012, MNRAS, 427, 1449

Lata S., Pandey A. K., Panwar Neelam et al. 2016 MNRAS, 456, 2505

Lata S., Pandey A. K., Yadav Ram Kesh et al. 2017, AJ, in preparation

Lata S., Yadav Ram Kesh, Pandey A. K. et al. 2014, MNRAS, 442, 273

Lata S. et al. 2017, in preparation

Lomb N. R. 1976, ApSS, 39, 447

Moskalik P., Dziembowski W. A. 1992, A\&A, 1992, 256, 5

Mowlavi N., Barblan F., Saesen S., Eyer L. 2013, A\&A, 554, 108

Pandey A. K., Sharma S., Ogura K. et al. 2008 , MNRAS , 383 , 1241

Robitaille T. P., Whitney B. A., Indebetouw R., Wood K. 2007, ApJS, 169 , 328

Scargle J. D. 1982, ApJ, 263, 835

Scholz A., Eisloffel J., Mundt R. 2009, MNRAS, 400, 1548

Siess L., Dufour E., Forestini M. 2000, A\&A, 358, 593

Sharma S., Pandey A. K., Ojha D. K. et al. 2007, MNRAS , 380 , 1141

Stankov A., Handler G. 2005, ApJS, 158, 193

Stetson P. B. 1987, PASP, 99, 191

Stetson P. B. 1992, J. R., Astron. Soc. Can., 86, 71 